\begin{tabular}{|c|c|}
\hline JUL 15 1996, ENGINEERING DATA TRANSMITTAL & 1. EDT NO 612368 \\
\hline
\end{tabular}

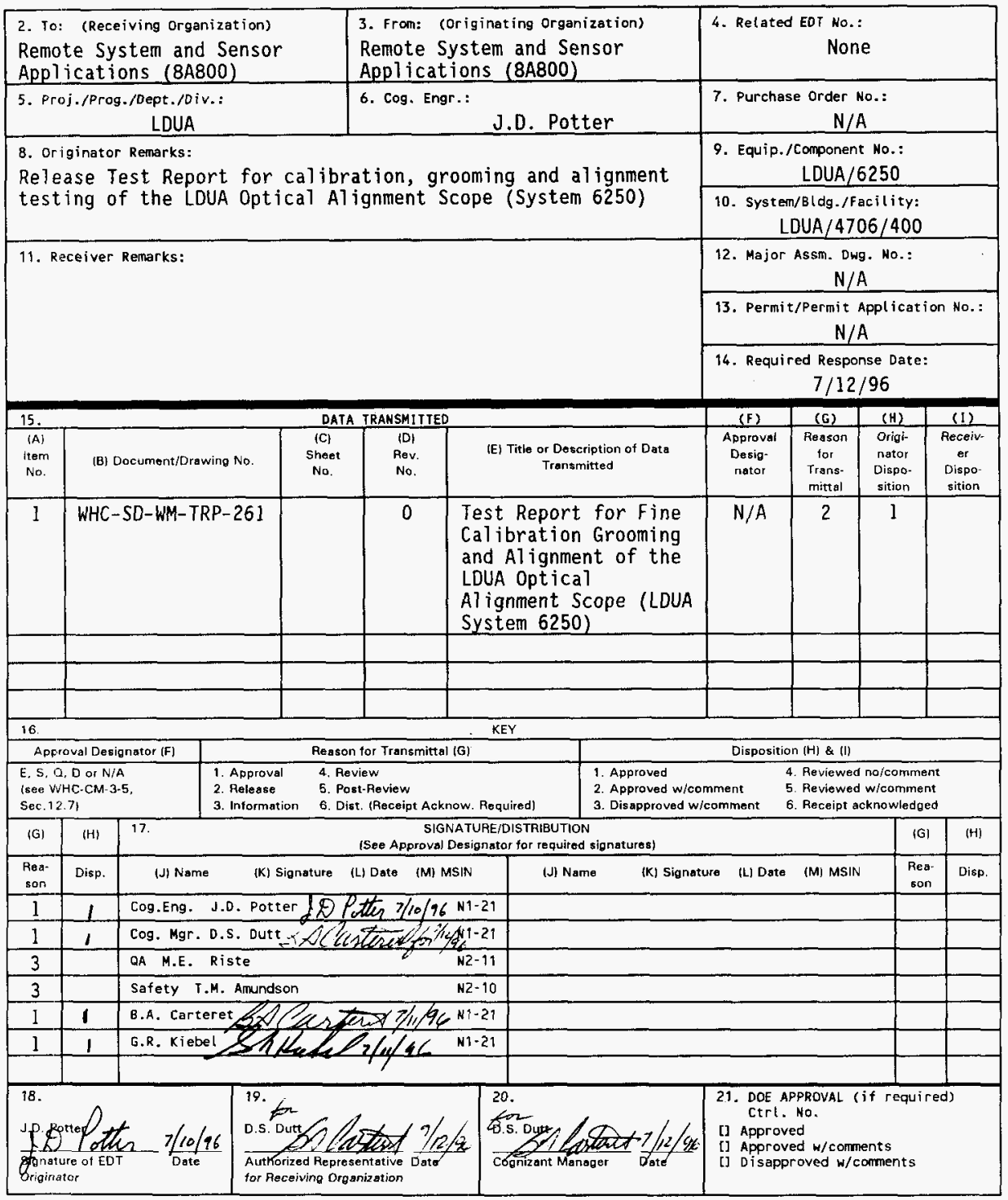

$B D-74(10-172-2(04 / 94)$ GEF097 


\title{
Test Report for Fine Calibration Grooming and Alignment of the LDUA Optical Alignment Scope (LDUA System 6250)
}

\author{
J.D. Potter \\ Westinghouse Hanford Company, Richland, WA 99352 \\ U.S. Department of Energy Contract DE-AC06-87RL10930
}

EDT/ECN: EDT $612368 \quad$ UC: 2060

Org Code: 8A800 Charge Code: HIE02

B\&R Code: EW4010000 Total Pages: 24

Key Words: LDUA, OAS, Alignment, Test, Report, Calibration, CG\&A

Abstract: The Light Duty Utility Arm (LDUA) is a remotely operated manipulator used to enter into underground waste tanks through one of the tank risers. The LDUA must be carefully aligned with the tank riser during the installation process. The Optical Alignment Scope (OAS) is used to determine when optimum alignment has been achieved between the LOUA and the riser. Calibration, grooming and alignment (CG\&A) is performed on the OAS to assure that the instrumentation and equipment comprising the OAS is properly adjusted in order to achieve its intended functions successfully. This document contains the results, conclusions and recommendations arrived at by the CG\&A tests performed on the OAS in accordance with WHC-SD-WM-TC-070.

TRADEMARK DISCLAIMER. Reference herein to any specific comercial product, process, or service by trade name, trademark, manufacturer, or otherwise, does not necessarily constitute or imply its endorsement, recommendation, or favoring by the United States Government or any ageney thereof or its contractors or subcontractors.

Printed in the United States of America. To obtain copies of this document, contact: WHC/BCS Document Control Services, P.O. BOK 1970, Mailstop H6-08, Richland WA 99352, Phone (509) 372-2420. Fax (509) 376-4989.
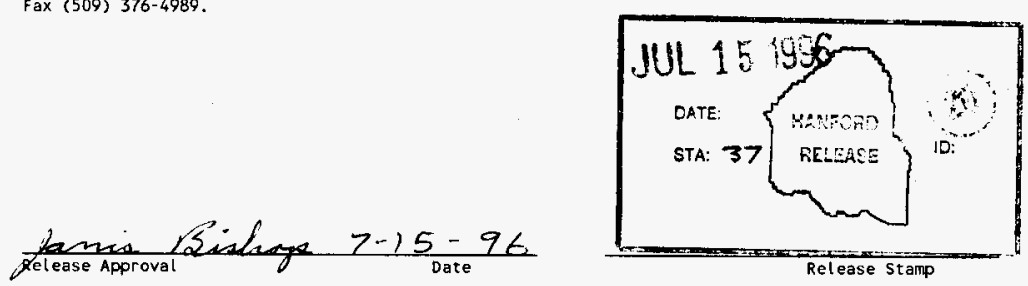

\section{Approved for Public Release}


WHC-SD-WM-TRP-261

Revision 0

\begin{abstract}
TEST REPORT
for

FINE CALIBRATION, GROONING AND ALIGNMENT

OF THE LDUA OPTICAL ALIGNMENT SCOPE
\end{abstract}

(LDUA System 6250)

July 10, 1996

by

J.D. POTTER

Remote System and Sensor Applications

Westinghouse Hanford Company

Richl and, Washington 
WHC-SD-WM-TRP-261

Revision 0

\section{TABLE OF CONTENTS}

Test Report for

Fine Calibration, Grooming and Alignment of the LDUA Optical Alignment Scope

Page Number

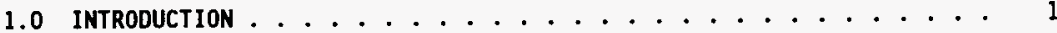

2.0 DESCRIPTION OF TEST ...................... 1

3.0 TEST METHOD AND TEST EqUIPHENT . . . . . . . . . . . . . . . 2

3.1 DESCRIPTION OF TEST METHODS . . . . . . . . . . . . . . 2

3.2 EQUIPMENT USED....................... . . . 2

4.0 TEST RESULTS . . . . . . . . . . . . . . . . . . . . 4

4.1 TEST RESULTS FOR OAS CONFIGURATION A . . . . . . . . . . 4

4.1.1 Wiring Verification Test (configuration A) . . . . . . 4

4.1.2 Pressure Switch Test (configuration A) . . . . . . . 4

4.1.3 OAS Alignment Fixture Installation (configuration A) , 4

4.1.4 Point Laser Adjustment (configuration A) . . . . . . 4

4.1.5 Video Camera Alignment (configuration A) . . . . . 5

4.1.6 Proximity Sensor Test (configuration A) . . . . . 5

4.2 TEST RESULTS FOR OAS CONFIGURATION B . . . . . . . . . . . 6

4.2.1 Wiring Verification Test (configuration B) . . . . . . 6

4.2.2 Pressure Switch Test (configuration B) ........ 6

4.2.3 OAS Alignment Fixture Installation (configuration B) - 6

4.2.4 Point Laser Adjustment (configuration B) . . . . . . . 6

4.2.5 Video Camera Alignment (configuration B) . . . . . . . 6

4.2.6 Proximity Sensor Test (configuration B) . . . . . . 7

4.3 MODIFICATIONS MADE TO THE OAS . . . . . . . . . . . . 8

4.3.1 OAS Configuration A Modifications . . . . . . . . . 8

4.3.2 OAS Configuration B Modifications .......... 8

4.4 ANOMALIES AND UNEXPECTED EVENTS . . . . . . . . . . . . . 9

5.0 CONCLUSIONS AND RECOMNENDATIONS . . . . . . . . . . . . . 10

6.0 DISPOSITION OF TEST ITEM . . . . . . . . . . . . . . . . . . . 11

7.0 REFERENCES .......................... 11 
WHC-SD-WM-TRP-261

Revision 0

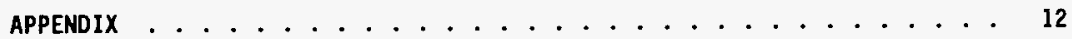

Figure 1, OAS Alignment Equipment Arrangement . . . . . . . 13

Figure 2, New Parts Used in the OAS ............... 14

Figure 3 , Location Designations for Proximity Sensors . . . . . . . . 15

Proximity Sensor Calibration Data (OAS Configuration A) . . . . . . 16

Proximity Sensor Calibration Data (OAS Configuration B) . . . . . 18 
WHC-SD-WM-TRP-261

Revision 0

\subsection{INTRODUCTION}

This report documents the results of the Component Grooming and Alignment (CG\&A) tests performed on the LDUA Optical Alignment Scope (OAS) as authorized in WHC-SD-TD-TP-005, revision 0, Light Duty Utility Arm System Pre-Operationa? (Cold Test) Test Plan. The CG\&A testing addressed in this report was performed in accordance with test procedure WHC-SD-WM-TC-070, revision 0 .

The OAS is attached to the LDUA wrist using an end effector Tool Interface Plate (TIP) in like manner as other LDUA end effectors. It utilizes a video camera and lights to allow the operator to align the LDUA mast with the tank riser using optical "bore scope" techniques, and four downward looking point laser lights to confirm that the riser walls do not protrude into the path. Before being inserted into the riser, the OAS will provide the operator with real-time information that will assist him/her in making a determination of how to move the LDUA mast for alignment. When the video camera and lasers indicate that the OAS is aligned to the riser, the LDUA mast and arm will also be in alignment with the riser (within the ability of the LDUA to correctly point and hold the OAS). Eight proximity sensing lasers around the circumference of the OAS allow the OAS to determine clearances between it and the wall of the riser as it subsequently passes through the riser.

The ability of the OAS to detect satisfactory LDUA alignment with a tank riser is directly related to how well the video camera and lasers are aligned relative to the central axis of the OAS. In the condition in which it was received from the supplier the OAS lasers and camera had not been aligned. Therefore, the objective of the CG\&A accomplished by this test was to adjust the video camera and downward looking point lasers as required to properly align them relative to the axis of the OAS, and to calibrate the side looking proximity sensors (also lasers) for proper distance readouts.

\subsection{DESCRIPTION OF TEST}

The OAS was designed and fabricated by Savannah River in accordance with requirements provided by WHC in WHC-SD-TD-FRD-003 (rev. 1), Functions and Requirements for the LDUA Integrated System, and WHC-SD-TD-CR-00I (rev. 1) Design Criteria for the Light Duty Utility Arm System End Effectors. Only one OAS exists (at this time), it was tested in two separate configurations (configurations $A$ and $B$ ). Configuration $A$ is the $O A S$ as it was received from Savannah River, with some modifications made by WHC after its arrival as listed in section 4.3.1. Configuration $A$ was tested first because no actual TIP was to be available for many weeks after the OAS arrived from Savannah River, yet there was much to be learned by testing the OAS while awaiting the TIP. Therefore, configuration $A$ was tested to detect any major problems as soon as possible. The test results for CG\&A of configuration $A$ are documented in the Interim Report in the appendix of this document.

After testing was complete on configuration $A$ it was made into configuration B by replacing its surrogate Tool Interface Plate (TIP) with an 
actual TIP and incorporation of a reticle generator into the video system. See section 4.3 .2 for a 1 ist of the changes made to the OAS in upgrading it to configuration $B$. Configuration $B$ is the final version of the OAS intended for actual use at the tank farms. All tests performed on configuration $A$ were repeated on configuration $B$. Testing was performed by WHC Remote Systems and Sensor Applications personnel, between February 6 to July 8, 1996.

\subsection{TEST METHOD AND TEST EQUIPMENT}

The CG\&A testing addressed in this report was performed as described in test procedure WHC-SD-WM- TC-070, revision 0 . Testing was done on the $12 \mathrm{ft}$ mezzanine of room 319 of the 427 building (FMEF) (a clean facility, with no history of exposure to radiological or toxic environments). A hole was made in the deck of the $12 \mathrm{ft}$ mezzanine per ECNs 611746 and 165800 , to enable visual access to the floor below. Final calibration of the eight proximity sensing lasers was done on the $42 \mathrm{ft}$ mezzanine of the FMEF with the OAS attached to the LDUA arm.

\subsection{DESCRIPTION OF TEST METHODS}

The alignment of the four downward looking point lasers and video camera was accomplished using special fixturing for the OAS and its video camera as shown schematically in figure 1. In general, testing consisted of using the fixture to support the OAS in a fixed frame of reference relative to known targets simulating a tank riser, then adjusting the video camera and point lasers to obtain a "standard" video image to be used as a reference image for future alignment tasks at the site. The alignment data obtained by this portion of the test is of a qualitative type which relies on the experience and visual acuity of the observer (i.e. the cognizant test engineer), to determine its acceptability.

The OAS has eight laser proximity sensors on its outer surface for detecting proximity of the riser wall. Each of these eight lasers was individually tested and calibrated using special calibration gages ( $\mathrm{H}-4-302540-1$ and -2$)$ designed for that purpose. Each calibration gage was attached in a fixed position over the sensor port in the body of the OAS to provide a surface which the sensor could detect at a known distance away. The sensor readout was corrected and compensated as required to produce the proper readout that should be obtained for the respective calibration gage.

\subsection{EQUIPNENT USED} testing:

The equipment listed below was used in the performance of the OAS CG\&A

- OAS Alignment Fixture, H-6-14330-1

- OAS Alignment Target, H-6-14337-1 
- Surrogate TIP (for OAS configuration A only), to enable OAS configuration $A$ to be attached to the OAS alignment fixture

- Camera test fixture (for OAS configuration B only)

- Camera alignment spacer and clamps (for OAS configuration B only), see figure 2

- Proximity sensor calibration gages, H-4-302540-1, and -2, Standards Code Numbers 635-10-01-001 and 635-10-01-002 respectively. Next calibration dates $1 / 31 / 99$.

- Master Precision Level, Starrett Model No. 1992 (sensitive to 0.0005 inches per foot). Standards Code Number 635-42-01-001. Next calibration date $12 / 8 / 96$.

- Plumb-bob and string, Starrett Model No. 870

- Multi-wire cable (i.e. test umbilical)

- Common Video End Effector (CVEE) Support Electronics, H-6-14270

- Test Power Supply

- TV monitor, Sony Model No. PVM-1350, S/N 2001122

- Video encoder/decoder, XYBion Model No. VED-100, S/N 15027 
WHC-SD-WM-TRP-261

Revision 0

\subsection{TEST RESULTS}

This section contains a brief description of each section of the test procedure and the results that were obtained with OAS configurations $A$ and $B$.

\subsection{TEST RESULTS FOR OAS CONFIGURATION A}

\subsubsection{Wiring Verification Test (configuration A)}

Section 11.1 of the procedure verified correct point-to-point routing of the electrical wiring from the device within the OAS to the applicable pin within the connectors at the TIP. The OAS end effector was disassembled and the wiring traced to make sure it matched the Savannah River drawings. Some corrections were noted and marked up drawings were sent back to Savannah River for incorporation. Before testing was completed the OAS was rewired by INEL. This rewiring was done to make it useable with the new Common Video End Effector (CVEE) control chassis.

\subsubsection{Pressure Switch Test (configuration A)}

Section 11.2, Pressure Switch Test, was deleted because the pressure switch was eliminated from the OAS due to a design change. The pressure switch sense port in the body of the OAS was welded closed and is no longer visible.

\subsubsection{OAS Alignment Fixture Installation (configuration A)}

Section 11.3 of the procedure installed the OAS Alignment Fixture (H-6-14330-10, OAS Alignment Target (H-6-14337-1) and the OAS in the test facility. Completion of this section verified the ability of the fixture to support the OAS and adequately achieve the required alignment between the fixture and the target on the floor. No problems were encountered.

\subsubsection{Point Laser Adjustment (configuration A)}

Section 11.4.1, Point Laser Alignment, was satisfactorily completed. Completion of this section verified the ability to adjust each of the four point lasers relative to the target on the floor. However, the quality of the "dot" image produced was found to be compromised by the optics of the OAS, but still useable. The dots were larger than desired, misshapen, and had poorly defined edges. Some lasers had multiple scattered secondary points of light several inches away from the real laser dot. 
WHC-S[-WM-TRP-26]

Revision 0

\subsubsection{Video Camera Alignment (configuration A)}

Section 11.4.2, Video Camera Alignment, was partially completed. The coarse positioning of the TV camera to achieve an image approximately centered on the monitor, was satisfactorily demonstrated. The dotted reticle generator (i.e. video encoder/decoder) was not available to allow the remaining portions of this section to be completed. A camera operation test was made to determine the visual acuity and low light level capabilities of the OAS video camera in detecting the images and patterns formed by the dot lasers along the wall of a simulated riser, and on a simulated waste surface under various lighting conditions (using only the DAS camera lights). This test was not in the CG\&A test procedure and was done informally for information only. The results were satisfactory.

\subsubsection{Proximity Sensor Test (configuration A)}

Section 11.5, Proximity Sensor Test, was satisfactorily completed. This section provided the calibration information necessary to enable the eight laser proximity sensors (four at top of OAS, four at bottom of OAS) to measure the radial distance between the OAS and the riser wall. For this test the OAS was lying on a table and readings were obtained locally without SDAS. See Table 1 in the appendix of this document for results obtained. 
WHC-SD-WM-TRP-261

Revision 0

\subsection{TEST RESULTS FOR OAS CONFIGURATION B}

\subsubsection{Wiring Verification Test (configuration B)}

Section 11.1 of the procedure verified correct point-to-point routing of the electrical wiring from the device within the OAS to the applicable pin within the connectors at the TIP.

\subsubsection{Pressure Switch Test (configuration B)}

DELETED (see text under section 4.1 .2 of this document)

\subsubsection{OAS Alignment Fixture Installation (configuration B)}

Section 11.3 of the procedure installed the OAS Alignment Fixture (H-6-14330-1, OAS Alignment Target $(H-6-14337-1)$ and the OAS in the test facility. The fixture was allowed to remain installed after configuration $A$ testing, and only required rechecking of dimensional parameters. No problems were encountered.

\subsubsection{Point Laser Adjustment (configuration B)}

Section 11.4.1, Point Laser Alignment, was completed as well as could be expected for the limited adjustment capabilities of the laser brackets and the poor quality of the laser image. (See remarks in section 4.1 .4 of this document)

\subsubsection{Video Camera Alignment (configuration B)}

Section 11.4.2, Video Camera Alignment, was completed as well as could be expected for the limited adjustment capabilities of the camera bracket. It was discovered that once the generated reticle was centered on the target, it would not remain centered when the camera lens was zoomed in or out. The lens of the camera was found to not be in the center of the OAS window. The threaded connection between the camera and its lens was not a tight fit and allowed significant play between them.

The camera was epoxyed to the lens to eliminate the play between them. A spacer (see figure 2 in appendix) was added to center the camera lens in the OAS window. Two clamps were added to allow the camera/lens assembly to be relocated to the center of the OAS window and to allow it to be angulated by adding shims beneath its mounting bracket if required (shims were not required). A camera test fixture (see figure 1 in appendix) was mounted to the base of the OAS alignment fixture to position a upper test ring at the same distance below the OAS as would be the upper end of a riser in the field. The target on the floor (simulating lower end of riser), the upper test ring (simulating upper end of a riser), and the OAS camera/lens assembly (bottom plate of OAS with camera, lens, lights, etc attached and leveled), were all made to be on the same vertical centerline as determined with a plumb-bob. The generated reticle was then made to be centered on the image of the target observed on the video monitor while zoomed such that the upper ring nearly 
filled the screen (becoming the "standard" zoom position). The generated reticie was at coordinates of $X=+.004, Y=.000$, to be on center of target (becoming the "standard" reticle position). A check using a transparent graduated overlay, centered on the generated reticle, found that the image of the upper ring and target were closely concentric (indicating that the camera/lens assembly was adequately in-line with the ring and target).

The lower portion of the OAS (with camera/lens assembly inside) was reattached to the upper portion of the OAS and the now complete OAS reattached to the OAS alignment fixture. The OAS fixture was also on the same centerline as the target, etc. as checked with a plumb-bob. After returning to the "standard" zoom, the generated reticle was set at its "standard" coordinates of $X=+.004, Y=.000$, and the ring and target images checked for concentricity using a transparent overlay as before. The observed images were again found to be closely concentric.

Each of the four point lasers were rechecked relative to the target on the floor and found to be acceptable and unchanged from their previous settings. The OAS is now fully assembled with its video camera and point lasers adequately aligned.

\subsubsection{Proximity Sensor Test (configuration B)}

Section 11.5, Proximity Sensor Test, was satisfactorily completed. This section provided the final calibration information necessary to enable the eight laser proximity sensors (four at top of OAS, four at bottom of OAS) to measure the radial distance between the OAS and the riser wall. For this test the OAS was attached to the end of the LDUA arm and gage blocks (H-4-302540-1 and -2 ) were used to provide known distances for comparison to sensor readings obtained in the Operations Control Trailer (OCT) using the SDAS. See Table 2 in the appendix of this document for results obtained. 
WHC-SD-WM-TRP-261

Revision 0

\subsection{MODIFICATIONS MADE TO THE OAS}

This section summarizes the changes that were made to the $O A S$ after it was received at Hanford from Savannah River.

\subsubsection{OAS Configuration A Modifications}

Before fine CG\&A testing of the OAS started, the following changes were incorporated:

1) The pressure switch was removed and the port was seal welded closed.

2) New brackets were made from a non-conductive material to hold the dot lasers.

3) Quartz windows were added in the body of the OAS to seal the port through which light from the dot lasers must pass.

4) A surrogate TIP was made, and attached to the OAS, to allow the OAS to be secured to the OAS alignment fixture.

5) The color TV camera was replaced with a black \& white camera for improved sensitivity in low light level applications.

6) The OAS was rewired by INEL. This rewiring was done to make it useable with the new Common Video End Effector (CVEE) control chassis. The old CVEE which was the Savannah River design, was worked on extensively but never functioned satisfactorily. The new INEL design replaced this one.

7) The TV camera reticle generator was not available, but will be available for later testing.

8) The RG187 coaxial cables for the video were epoxyed into the Hypertronic connector. The cable was continually breaking and this solved the problem.

\subsubsection{OAS Configuration B Modifications}

After testing was completed on OAS configuration A, OAS configuration B was made from $O A S$ configuration $A$ by performing the following modifications:

1) The surrogate TIP was removed and replaced with an actual end effector TIP provided by SPAR.

2) A spacer ring was added between the lens housing and the OAS end plate to center the lens within the end plate (see figure 2 in the appendix).

3) The fasteners securing the camera/lens assembly bracket to the OAS end plate were removed to enable the assembly to brought to the 
center of the end plate. Two clamps were added, one at each end of the bracket, to secure the bracket in its new location (see figure 2 in the appendix).

4) The camera was epoxyed to the lens to eliminate the play between them and to assure that they remain in proper adjustment.

5) The electronics supporting the OAS was revised to include a video encoder/decoder which can superimpose a small dotted reticle on the image observed on the monitor. The coordinates of this reticle can be varied, allowing it to be located in various positions on the screen in an accurate repeatable manner.

\subsection{ANOMALIES AND UNEXPECTED EVENTS}

While an actual end effector TIP was temporarily available at Hanford, a fit check was made between the TIP and the OAS and found to be satisfactory. A fit check was also made between the TIP and the OAS alignment fixture (similar to arm half TIP). Two of the three engagement pins of the TIP seemed to grip the fixture properly, with the pins lifting off from the actuating cam as they should. The third pin did not seem to lift off from the actuating cam. The pin in question was the pin counter-clockwise from the index pin, viewed looking from the arm half TIP.

The OAS alignment fixture has an interface with the OAS which is very much like an LDUA TIP. In one instance, when engaging the fully assembled OAS configuration B to the OAS alignment fixture, the OAS was lifted into position against the fixture and the three locking cams of the end effector TIP were rotated clockwise until they firmly bottom out, as is normal. Upon removing the support from the OAS it was obvious that the TIP was not engaged with the fixture, slipping out of the fixture and nearly tipping over. No damage was done and no one was injured. This event was discussed with those responsible for mating end effectors to the LDUA arm inside the TRIC, and techniques will be incorporated to assure that the tiwo TIP halves are fully secured before their support is removed. 
WHC-S[1-WM-TRP-26]

Revision 0

\subsection{CONCLUSIONS AND RECOMMENDATIONS}

In general, the OAS and the OAS alignment fixture seemed to be adequate for accomplishing their intended purposes. The most significant areas in which improvements are recommended to be made in future generations of OAS are:

1) Provisions should be made to enable positional adjustments to be made to the video camera/lens assembly, and to each of the four point lasers, while the OAS is fully assembied. The brackets which hold the dot lasers and the camera/lens assembiy do not have means of adjustment, making the alignment of these devices difficult. The current OAS design requires the OAS to be partially disassembled when making these adjustments, and shimming to get the devices in proper position. The alignment of the camera/lens assembly is mostly trial and error. These tasks were difficult to do in a clean, shirt-sleeve environment, and would be extremely difficult (or impossible) to do wearing protective clothing and gloves.

2) The camera/lens assembly should have adjustments ( $X$, $Y$, pitch and roll) to enable it to be accurately positioned on, and aligned with, the center axis of the OAS. These adjustments should be capable of being performed without having to disassemble the OAS (see item l above). This would allow the reticle generated by the encoder/decoder to remain on target as the lens is zoomed in and out, thereby providing better alignment with the target during CG\&A. More accuracy in measuring concentricity could also be obtained by zooming in to enlarge the image without inducing error.

3) The quality of the image generated by each of the dot lasers was not as good as expected for this class of instrument, and improvements should be diligently sought after in the next generation of OAS. The problem is primarily attributed to distortion caused by the quartz windows and the prisms, but there could also be some other contributing factors (edge of small hole in body of OAS interfering with light beam, etc). As it now is the laser beams are still believed adequate for determining which way to translate the LDUA MDS $X-Y$ table during an actual alignment operation, although much more operator judgment may be required to interpret the laser traces within the riser, especially for tight fitting risers.

4) The reticle generated by the encoder/decoder used in this test was too small to fully extend across the monitor screen, and could not be used by itself to assist in determining concentricity of the upper and lower riser end. The existing encoder/decoder is only useful as a reference for locating a transparent overlay of a larger graduated reticle on the monitor screen. Such a transparent overlay is difficult to use with accurate results because of the parallax due to the distance between the overlay and the image observed on the monitor CRT. An improved encoder/decoder should be included in the OAS support electronics which would provide a larger computer generated image of a reticle with graduated marks directly imposed on the observed riser images. This would eliminate the need for a transparent overlay over the monitor and provide more accurate readings without inducing error due to parallax. 


\subsection{DISPOSITION OF TEST ITEM}

Having successfully completed the tests described in this document, the OAS will be made available to support other LDUA cold tests (e.g. acceptance testing, qualification testing, operation training, etc.) to be conducted in the 427 building (FMEF). After all cold testing has been completed the OAS will be made available at tank farms for hot testing in an actual waste tank.

\subsection{REFERENCES}

- WHC-SD-WM-TC-070, revision 0, Test Procedure for CG\&A of the LDUA Optical ATignment Scope

- WHC-SD-TD-TP-005, revision 0, Light Duty Utility Arm System Pre-0perational (Cold Test) Test Plan

- WHC-SD-TD-FRD-003, revision 1, Functions and Requirements for the LDUA Integrated System

- WHC-SD-TD-CR-001, revision 1, Design Criteria for the LDUA System End Effectors

- ECN 611746 (facility modifications)

- $\quad$ ECN 165800 (facility modifications)

- H-6-14330, revision 0, OAS Alignment Fixture Final Assembly

- H-6-14337, revision 0, LDUA OAS Target Plate Assembly

- H-4-302540, revision 0, OAS Calibration Gauge Details

- H-6-14320, revision A, (Savannah River, EES-22408-R1-021), TIP Mounted Systems Alignment End Effector Assembly 
WHC-SD-WM-TRP-261

Revision 0

APPENDIX 
WHC-SD-WM-TRP-261

Revision 0

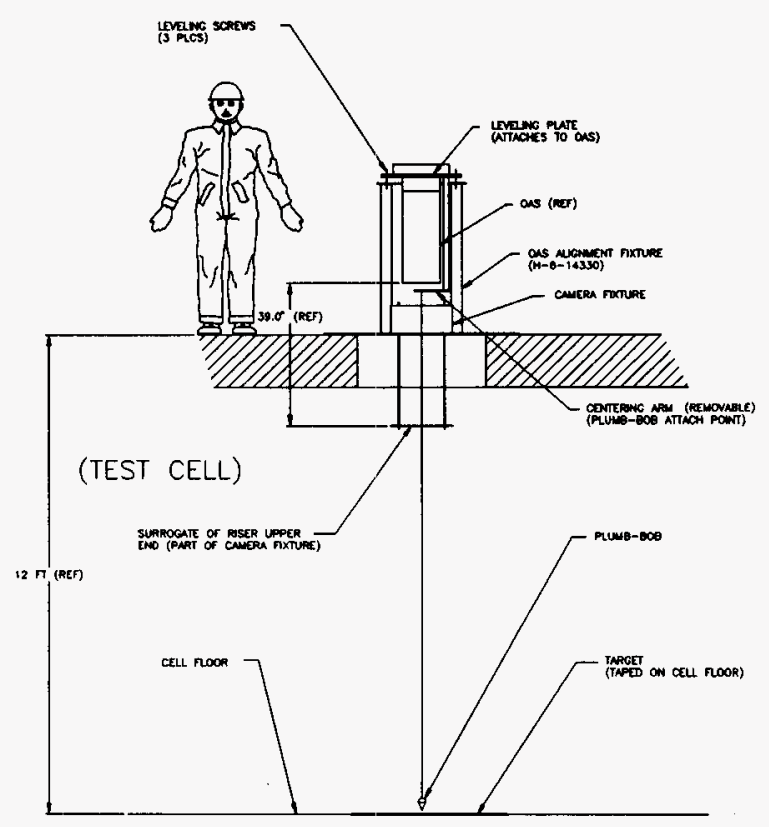

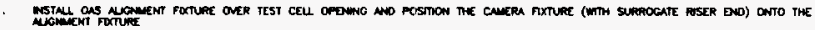

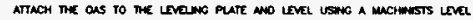

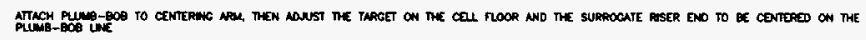

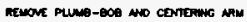

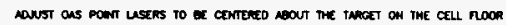

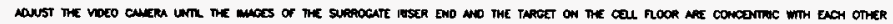

Figure 1

OAS Alignment Equipment Arrangement 
WHC-S[I-WM-TRP-26]

Revision 0
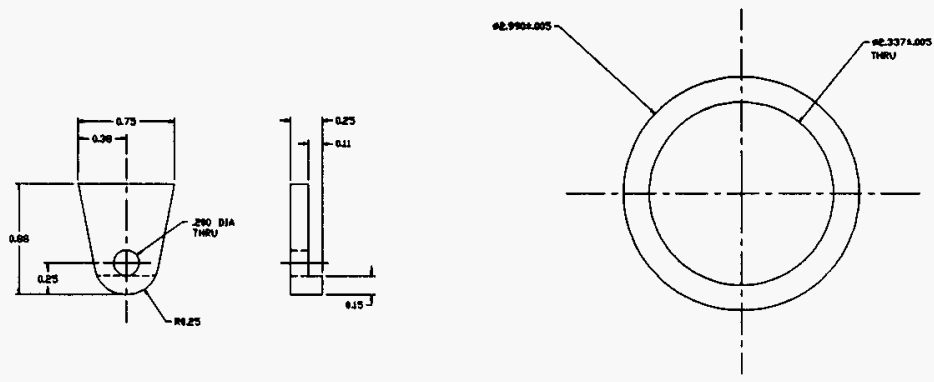

CLAMP, LENS BRACKET (2 ReOp) mitro mumumem

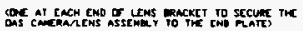

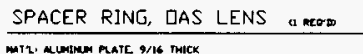

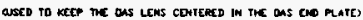

Figure 2

New parts used in the OAS 
WHC-SD-WM-TRP-261

Revision 0

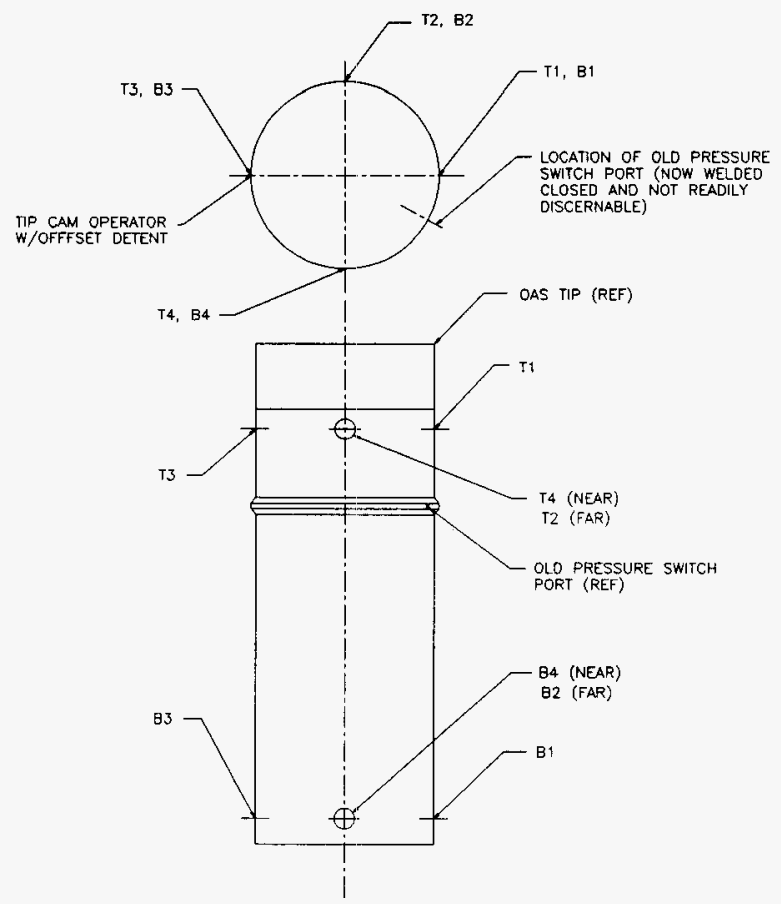

Figure 3

Location Designations for Proximity Sensors 
WHC-SD-WM-TRP-261

Revision 0

\section{Proximity Sensor Calibration Data (OAS configuration A)}

The following data was obtained from the eight OAS proximity sensing lasers (see figure 3 for location of each respective laser):

\begin{tabular}{|c|l|l|l|l|l|l|l|l|}
\hline \multirow{2}{*}{$\begin{array}{l}\text { Gage } \\
\text { Block } \\
\text { used: }\end{array}$} & \multicolumn{8}{|c|}{ Sensor Location Number (see fig.3) } \\
\cline { 2 - 9 } & B1 & B2 & B3 & B4 & T1 & T2 & T3 & T4 \\
\hline-1 & 2.75 & 2.72 & 2.80 & 2.76 & 2.72 & 2.70 & 2.74 & 2.74 \\
\hline-2 & 2.58 & 2.55 & 2.63 & 2.60 & 2.55 & 2.54 & 2.57 & 2.57 \\
\hline
\end{tabular}

The above data obtained from the proximity sensors is the total light path distance " $L "$ in inches from the sensor, to the mirror, to the gage block surface as shown in the sketch below.

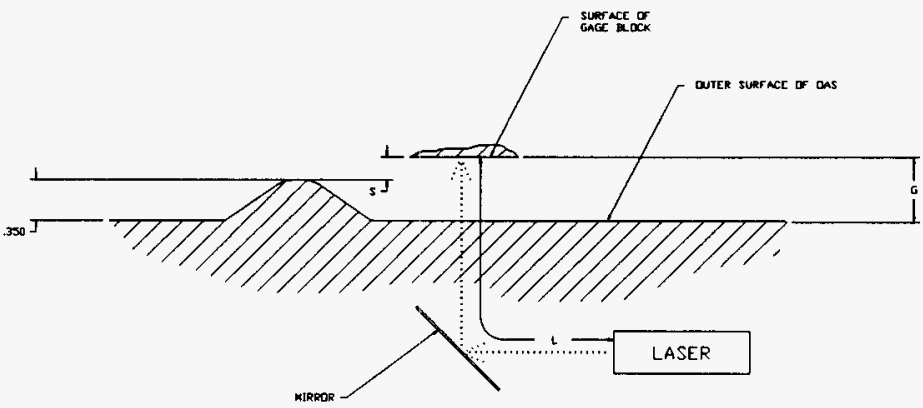

Where: $L=$ Total distance in inches from the sensor, to the mirror, to the gage block surface

$G=$ Known distance from gage block surface to cylindrical portion of OAS body (.545" for block -1 , and .380" for block -2)

$S=$ Known distance between gage block surface and the prism housing of the OAS $(=G-.350) \quad S_{1}=.195$ for gage block -1 , and $S_{2}=.030$ for gage block -2

(continued next page) 
OAS configuration A was not setup to provide a direct readout of the distance "S" from the prism housing of the OAS to the gage block. Instead, a constant "C" was determined which could be subtracted from the sensor readings to provide "S" (i.e. $S=L-C$ ). The constant " $C$ " used for each sensor was the average of the values of " $C$ " calculated using the observed values of " $L$ " obtained with the two gage blocks. For example:

for sensor Bl

using gage block $-1, L_{1}$ was observed to be $2.75^{\prime \prime}$ using gage block $-2, L_{2}$ was observed to be 2.58 "

$C_{1}=L_{1}-S_{1}=2.75-.195=2.555$

and

$\mathrm{C}_{2}=\mathrm{L}_{2}-\mathrm{S}_{2}=2.58-.030=2.55$

and the average value of $C$ is calculated to be:

$C=\left(C_{1}+C_{2}\right) / 2=(2.555+2.55) / 2=2.553$ (inches)

Below is a summary showing the constant $C$ for each sensor:

\begin{tabular}{||c|c|c|c|c|c|c|c|c||}
\hline & \multicolumn{7}{|c|}{ Sensor Location Number (see figure 3) } \\
\cline { 2 - 10 } & $\mathrm{B} 1$ & $\mathrm{~B} 2$ & $\mathrm{~B} 3$ & $\mathrm{~B} 4$ & $\mathrm{~T} 1$ & $\mathrm{~T} 2$ & $\mathrm{~T} 3$ & $\mathrm{~T} 4$ \\
\hline $\mathrm{C}_{1}$ & 2.555 & 2.525 & 2.605 & 2.565 & 2.525 & 2.505 & 2.545 & 2.545 \\
\hline $\mathrm{C}_{2}$ & 2.55 & 2.52 & 2.60 & 2.57 & 2.52 & 2.51 & 2.54 & 2.54 \\
\hline $\mathrm{C}$ & 2.553 & 2.523 & 2.603 & 2.568 & 2.523 & 2.508 & 2.543 & 2.543 \\
\hline
\end{tabular}




\section{Proximity Sensor Calibration Data (OAS configuration B)}

The following data was obtained from the eight OAS proximity sensing lasers while the OAS was attached to the LDUA and readings obtained by SDAS in the Operation Control Trailer (OCT) (see figure 3 for location of each respective laser):

\begin{tabular}{||c|c|c|c|c|c|c|c|c||}
\hline \hline \multirow{2}{*}{$\begin{array}{l}\text { Gage } \\
\text { Block } \\
\text { used: }\end{array}$} & \multicolumn{7}{|c|}{ Sensor Location Number (see fig.3) } \\
\cline { 2 - 10 } & B1 & B2 & B3 & B4 & T1 & T2 & T3 & T4 \\
\hline-1 & 2.76 & 2.69 & 2.80 & 2.75 & 2.72 & 2.72 & 2.76 & 2.74 \\
\hline-2 & 2.59 & 2.53 & 2.62 & 2.58 & 2.55 & 2.56 & 2.58 & 2.57 \\
\hline-1 & 2.77 & 2.69 & 2.80 & 2.73 & 2.72 & 2.71 & 2.75 & 2.73 \\
\hline-2 & 2.59 & 2.51 & 2.63 & 2.57 & 2.54 & 2.54 & 2.58 & 2.56 \\
\hline-1 & 2.76 & 2.68 & 2.79 & 2.74 & 2.72 & 2.72 & 2.76 & 2.73 \\
\hline-2 & 2.59 & 2.52 & 2.62 & 2.58 & 2.54 & 2.53 & 2.58 & 2.57 \\
\hline
\end{tabular}

The above data obtained from the proximity sensors is the total light path distance "L" in inches from the sensor, to the mirror, to the gage block surface as shown in the sketch below. The differences between OAS configuration $A$ and $B$ data is attributed to minor irregularities in the OAS body in the zone beneath the feet of the gage blocks, and to the fact that at least one sensor was disturbed in the interim. The data to be utilized for final OAS purposes is that obtained using OAS configuration B.

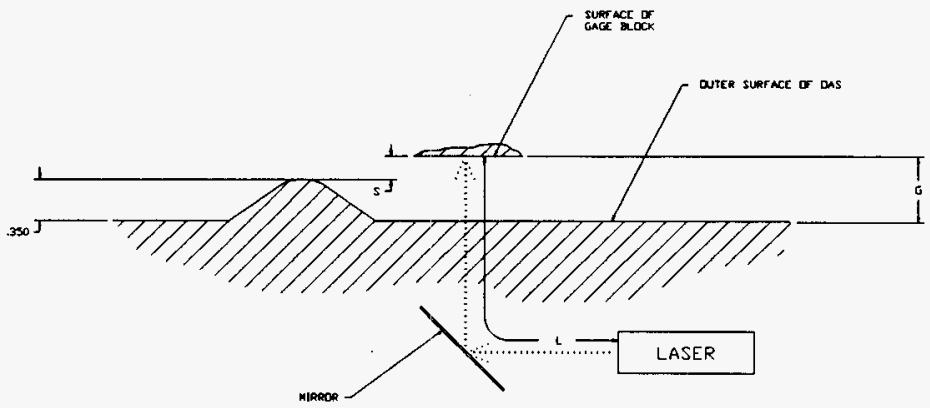


Where: $L=$ Total distance in inches from the sensor, to the mirror, to the gage block surface

$G=$ Known distance from gage block surface to cylindrical portion of OAS body (.545" for block -1 , and .380" for block -2 )

$S=$ Known distance between gage block surface and the prism housing of the OAS (=G-.350) $S_{1}=.195$ for gage block -1 , and $S_{2}=.030$ for gage block -2

The distance " $S$ " from the prism housing of the OAS to the gage block was used to determine a constant " $C$ " which could thereafter be subtracted from the sensor readings to provide "S" (i.e. $S=L-C$ ). The constant " $C$ " used for each sensor was the average of the values of " $C$ " calculated using the average of the three observed values of "L" obtained with the two gage blocks. For example:

for sensor Bl

using gage block $-1, L$, was observed to be $2.76,2.77$, and 2.76 or: $L_{1 \text { (avg) }}=2.763^{\prime \prime}$

using gage block $-2, L_{2}$ was observed to be $2.590,2.590$, and 2.590 or: $L_{2 \text { (avg) }}=2.590$

$C_{1}=L_{1 \text { (avg) }}-S_{1}=2.763-.195=2.568$

$\mathrm{C}_{2}=\mathrm{L}_{2 \text { (avg) }}-\mathrm{S}_{2}=2.590-.030=2.560$

and the average value of $C$ is calculated to be:

$C=\left(C_{1}+C_{2}\right) / 2=(2.568+2.560) / 2=2.564$ (inches)

Below is a summary showing the constant $C$ for each sensor:

\begin{tabular}{||c|c|c|c|c|c|c|c|c||}
\hline & \multicolumn{7}{|c|}{ Sensor Location Number (see figure 3) } \\
\cline { 2 - 10 } & $\mathrm{B} 1$ & $\mathrm{~B} 2$ & $\mathrm{~B} 3$ & $\mathrm{~B} 4$ & $\mathrm{~T}$ & $\mathrm{~T} 2$ & $\mathrm{~T} 3$ & $\mathrm{~T} 4$ \\
\hline $\mathrm{C}_{1}$ & 2.568 & 2.492 & 2.602 & 2.545 & 2.525 & 2.522 & 2.562 & 2.538 \\
\hline $\mathrm{C}_{2}$ & 2.560 & 2.490 & 2.593 & 2.547 & 2.513 & 2.513 & 2.550 & 2.537 \\
\hline $\mathrm{C}_{\mathrm{yyyyyyyy}}$ & 2.564 & 2.491 & 2.597 & 2.546 & 2.519 & 2.518 & 2.556 & 2.538 \\
\hline
\end{tabular}

The constant "C" from the above table will be automatically subtracted from the respective sensor reading, and the resulting difference " $S$ " will be displayed in the OCT as the distance between the OAS and the riser wall at that sensor location. Using the data from the sensors the operator may 
evaluate what the distance between the OAS and the riser walls is at points between sensor locations.

The sensor readings displayed in the OCT are in reference to the sensors located at the TIP end and the front end of the OAS, with circumferential designations of top, bottom, left, and right. The correlation between the data displayed in the OCT and the physical location (as shown in figure 3) of the sensors on the OAS are as tabulated below:

\begin{tabular}{l|ll|l} 
TIP top & T3 & front top & B3 \\
TIP bottom & T1 & front bottom & B1 \\
TIP right & T2 & front right & B2 \\
TIP left & T4 & front left & B4
\end{tabular}




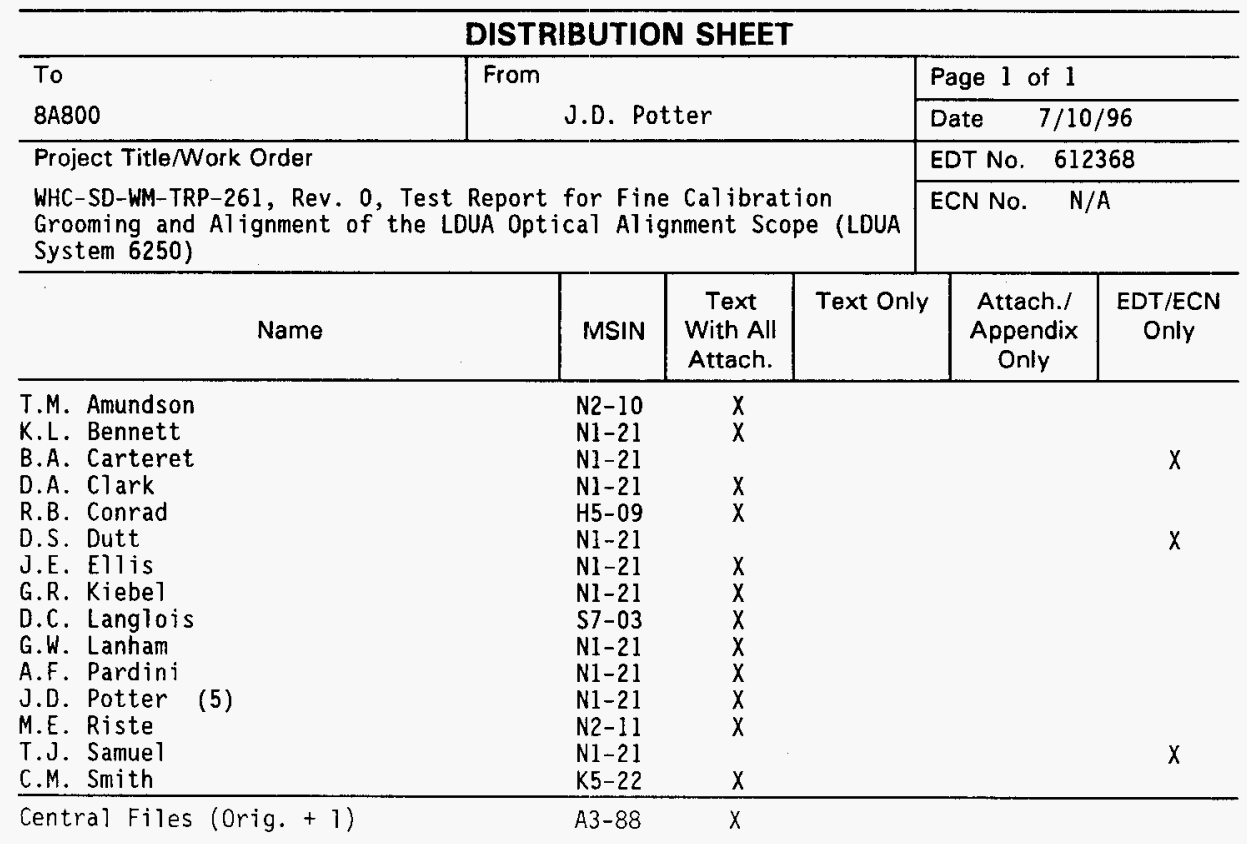

